\title{
Chronic periodontitis as an etiology of sleep disturbances and premenstrual syndrome (PMS)
}

\author{
Haryono Utomo \\ Department of Oral Biology \\ Faculty of Dentistry Airlangga University \\ Surabaya - Indonesia
}

\begin{abstract}
It is obvious that sleep disturbances may induced by acute pulpal or periodontal pain. Other causes of sleep disturbances which also termed as sleep dysfunction, or insomnia, according to the patient has to be treated by physician. Nevertheless, in a case report, surprisingly, periodontal treatment relieved sleep disturbances and premenstrual syndrome (PMS). Coincidentally, women also more vulnerable to sleep disturbances and periodontal disease. It is also interesting that the exact etiology of PMS is still unknown, and $80 \%$ women who suffered from PMS also experience sleep disturbances. Recently, there has been increasing numbers of literatures and evidence-based cases linking periodontal disease to systemic diseases. However, systemic effects of periodontal disease that lead to PMS which associated with sleep disturbances are rarely discussed. Several mechanisms had been proposed to involve in these symptoms: female sexual hormonal imbalance, stimulation of the hypothalamic-pituitary-adrenal axis (HPA-axis) and neurogenic switching mechanism. In addition, as estrogen makes women more susceptible to stress, it worsen the symptoms. The glucocorticoid hormones synthesized upon stimulation of the HPA-axis, either by stress or pro-inflammatory cytokines, may disrupt the sleep-wake cycle; and also create estrogen dominance. The aim of this study is to propose the etiopathogenesis of PMS which associated with sleep disturbances that may be related to chronic periodontitis. Since in this case report scaling and curettage resulted in the disappearing of PMS and sleep disturbances; the conclusion is that chronic periodontal disease may act as one of the etiologies of PMS and sleep disturbance.
\end{abstract}

Key words: Chronic periodontitis, Sleep disturbances, Premenstrual syndrome

Correspondence: Haryono Utomo, c/o: Bagian Biologi Oral, Fakultas Kedokteran Gigi Universitas Airlangga. Jln. Mayjend. Prof. Dr. Moestopo 47 Surabaya 60132, Indonesia. E-mail: dhoetomo@indo.net.id. Telp. 5053195

\section{INTRODUCTION}

What is a good sleep? A study revealed that sleeping shorter that 6 hours and more than 9 hours a day for adults is considered unhealthy, one of the risk factor is diabetes. The best time to sleep is at night, if nighttime sleep disturbances exists and daytime sleep or take a nap is needed, it should be less than 30 minutes, otherwise the circadian rhythm will be altered. ${ }^{1}$ In daily general medicine practice, most frequently reported complaints which affecting the quality of life by women is insomnia, a kind of sleep disturbance. It is interesting that as many as $80 \%$ of women report premenstrual syndrome (PMS) is associated with insomnia; in contrast, generally premenstrual insomnia disappears a few days after menstruation begins., ${ }^{2,3}$

Nevertheless, recent publications revealed that the exact etiology of PMS is still unknown; as the result, in insomnia related to PMS patients, the drug of choice is anti insomniacs i.e., hypnotics, anti-depressant. ${ }^{3}$ If the sleep disturbances are predicted to be one of PMS symptoms, the medications are mostly hormonal, i.e. gonadotropinreleasing hormone agonist; another drug of choice are cyclooxygenase-2 (COX2) inhibitors. Other treatments are biofeedback, massage and chiropractic. ${ }^{4}$ Unfortunately, dental and periodontal treatments are not included in treatment protocol for insomnia and PMS.

Compare to men, women are twice as likely to have difficulties in falling asleep or maintaining sleep at night, so called sleep disturbance. Sleep disturbance is also termed as sleep dysfunction, sleep disorders or insomnia. Nevertheless, sleep is sounder and less prone to disturbances during young adulthood; however, some women are prone to sleep problems during their reproductive years (30-40 years). Hormonal fluctuations associated with menstrual cycle and pregnancy may affect circadian rhythms and stress reactivity, thereby active women are more vulnerable to emotional stress and to concomitant sleep disturbances. ${ }^{2}$ It is also interesting that based on literatures, women are not only susceptible to stress, ${ }^{5}$ and debilitating symptoms (i.e. pain, ${ }^{6}$ sleep disturbances, ${ }^{2,3}$ chronic fatigue syndrome ${ }^{7}$ ), but also to periodontal disease. ${ }^{8}$

Recently, the role of periodontal disease as a source of focal infection that may initiate, perpetuate or aggravate the symptoms of systemic diseases such as cerebrovascular disease, cardiovascular disease, pregnancy problems and respiratory problems had already been recognized. ${ }^{9}$ Nevertheless, the relationship between sleep disturbances and chronic periodontitis is not clearly understood. 
A possible correlation between sleep disturbances and chronic periodontitis could be predicted regarding to a case report related to a female patient who suffered from sleep disturbance, symptoms mimicking PMS and dysmenorrhoe. After periodontal treatment had been done, headache disappeared instantly and she had an undisturbed sleep at the same night. In her next menstrual period, PMS and dysmenorrhoe did not reappear. ${ }^{10}$

However, the etiopathogenesis of the sleep disturbance associated with PMS in women which may induce by chronic periodontitis is still unclear. Researches should be done to find out an established explanation how untreated periodontitis may lead to sleep disturbances and PMS. If periodontal treatments are included in the treatment protocols, it will give a positive value to the sufferers, at least by avoiding unnecessary medications. The objective of this study is to propose the possibility of chronic periodontitis as an etiology of PMS which associated with sleep disturbances based on literatures review.

\section{Female sexual hormones and stages of menstrual period}

Since puberty, the role of sexual hormones is important in body system regulation. Estrogen and progestin are the most important female hormones. There are three estrogens present in significant quantities in the plasma of human female: estrone (E1), 17- $\beta$ estradiol (E2), and estriol (E3). The estrogenic potency of $17-\beta$ estradiol is 12 times that of estrone and 80 times of estriol. By far, the most important hormone of the progestins is progesterone. ${ }^{11}$

There are several stages of menstrual period after puberty: (1) Child bearing age (until 45 years old), (2) Peri-menopausal period (45-50 years) and (3) Menopausal periods (50 years and beyond). ${ }^{12}$ During peri-menopause there is a steep decrease of progesterone with a gradual decrease in estrogen. At this period women usually experience several uncomfortable symptoms including sleep disturbance or insomnia that are attributed to vasomotor symptoms (e.g., hot flushes and night sweats) rather than to menopausal status. ${ }^{2}$

\section{The premenstrual syndrome (PMS)}

Up to 85 percent of menstruating women report having PMS, and 2 to 10 percent report disabling, incapacitating symptoms. Symptoms typically begin between the ages of 25 and 35 years. ${ }^{12}$ Premenstrual syndrome is a recurrent luteal phase condition characterized by physical, psychological, and behavioral changes of sufficient severity to result in deterioration of interpersonal relationships and normal activity. ${ }^{4}$ There are many symptoms related to PMS such as: irritability, insomnia, depression, severe fatigue, headache, vertigo, syncope, visual disturbances, heart palpitation, numbness, hyperalgesia of arms and leg, allergy and infection. ${ }^{12,13}$

The exact etiology of PMS is still unknown, nevertheless PMS seems to be related to fluctuations in estrogen and progesterone. ${ }^{4,12}$ The following has been suggested as possible causes of PMS: estrogen-progesterone imbalance; excessive aldosterone, or ADH (hormone that functions in the regulation of the metabolism of sodium, chloride, and potassium); carbohydrate metabolism changes; hypoglycemia; allergy to progesterone; retention of sodium and water by the kidneys and psychogenic factors. ${ }^{13}$ Other factor is the raise of body temperature during the luteal phase mediated by increasing progesterone level. ${ }^{14}$

\section{Effects of female sexual hormones to the periodontium}

Estrogen and progesterone has disadvantageous effects to gingiva, as they may cause the susceptibility of women to periodontal disease Estrogen decreases gingival keratinization, whereas progesterone increases vasodilatation and permeability. Additionally, estrogen and progesterone may act as growth factors of prevotella intermedia that is Gram negative periodontopathogenic bacteria. ${ }^{8}$

\section{Estrogen in inflammation and immunity}

Estrogen has several role in inflammation, depends on the level. At low or physiological level, estrogen increases pro-inflammatory cytokine production interleukin-1 (IL-1), tumor necrosis factor- $\alpha$ (TNF- $\alpha$ ) and IL-6 by monocytes. ${ }^{15}$ Other effect of estrogen in low or physiological level is polarization of the $\mathrm{CD}^{+}{ }^{+}$(T-helper, Th) cells to the Th1 cells expression, through the stimulation of cytokines IL-12, TNF- $\alpha$ and IFN- $\gamma$ upon lipopolysaccharides (LPS) activation of antigen presenting cells (APC). Interferon- $\gamma$, a Th1 cytokine, stimulates macrophages or monocytes proliferation and activity. Conversely, high estrogen as in pregnancy, stimulate the Th2 cytokines (IL-4, IL-10 and IL-13) which inactivate macrophages. ${ }^{11,16}$

Many stress-related mental illnesses, including depression and post-traumatic stress disorder (PTSD), occur at least twice as often in women as in men. Depression and PTSD are characterized by the dysfunction of an area of the brain called the prefrontal cortex (PFC), which is known to govern higher cognitive abilities like level and shortterm memory. ${ }^{5}$ A better understanding of such processes may help to elucidate the reason why women are more susceptible to stress-related disorders. In an experiment, after exposure to higher levels of stress, both males and females made significant memory errors. However, after exposure to a moderate level of stress, females were impaired, but males were not, suggesting that females were more sensitive to the PFC-impairing effects of stress. In this study, ovariectomized females showed increased stress sensitivity only after estrogen replacement ${ }^{8,17}$

\section{The relationship between progesterone and cortisol}

Cortisol, a glucocorticoid hormone is a derivate of progesterone (Figure 1), ${ }^{16}$ and also able to compete between each other which termed the competitive binding mechanism. It is not surprising because progesterone binds to the human mineralocorticoid receptors (hMRs) with nearly the same affinity as cortisol. ${ }^{18}$ As the result, higher cortisol level which caused by stressor or pro-inflammatory cytokines may bind to the progesterone receptors which then lowers the progesterone level. One of the side effects 


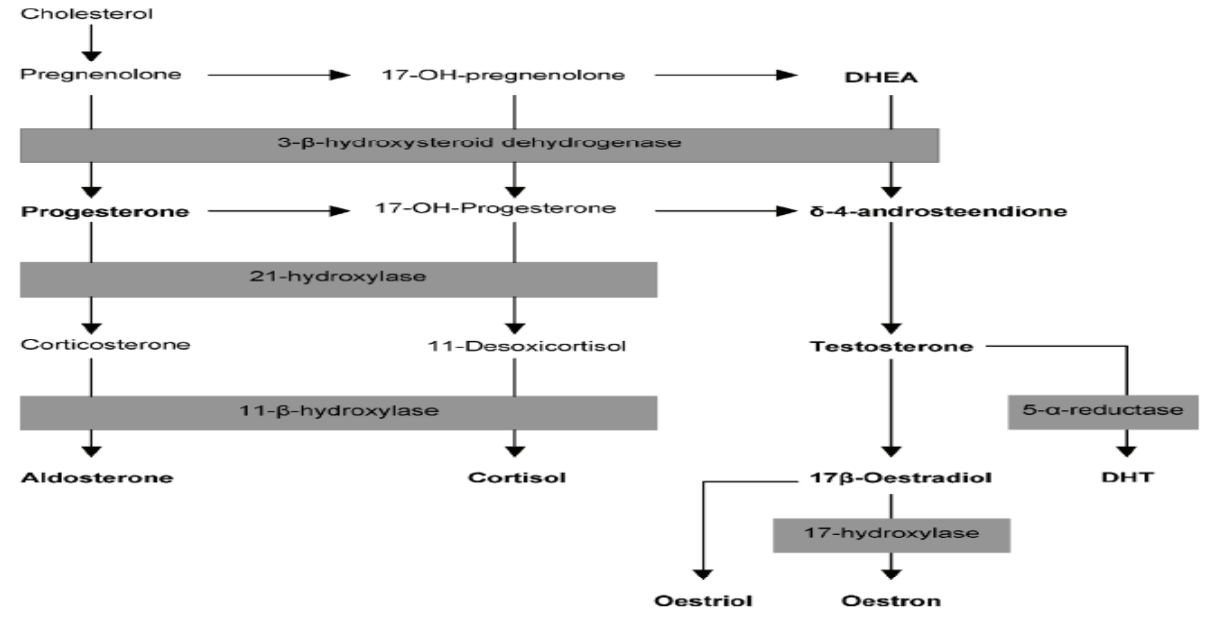

Figure 1. Biosynthesis of steroid hormones. ${ }^{16}$

of low progesterone level is high physiologic estrogen level which leads to estrogen dominance. ${ }^{19}$

\section{Estrogen dominance}

Estrogen and progesterone work in synchronization with each other as checks and balances to achieve hormonal harmony in both sexes. If estrogen level is relatively higher than normal to progesterone or it is rather the relative dominance of estrogen and relative deficiency of progesterone, it creates the condition that termed as estrogen dominance. Symptoms that exist in estrogen dominance are almost the same to PMS ${ }^{13,19}$

\section{The sleep - wake cycle and sleep disturbances}

Individuals differ considerably in their natural sleep patterns. Children and adolescents sleep more than adults, and young adults sleep more than older ones. Normal sleep consists of four to six behaviorally and electroence phalographically (EEG) defined cycles, including periods during which the brain is active (associated with rapid eye movements, called REM sleep), preceded by four progressively deeper, quieter sleep stages graded 1 to 4 on the basis of increasingly slow EEG patterns. Stages 1 and 2 are considered as light sleep; stages 3 and 4 are deep sleep or slow wave sleep (SWS) which gradually lessens

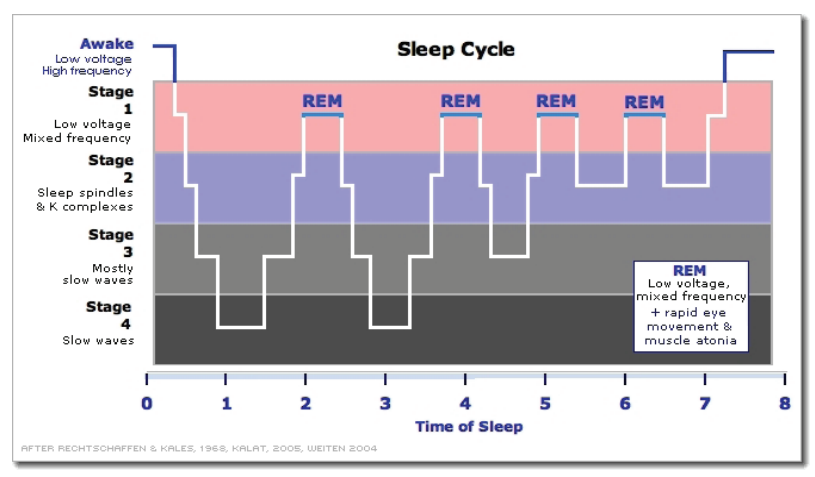

Figure 2. Stages of the sleep-wake cycle. ${ }^{22}$ with age and usually disappears in the elderly. Rapid eye movement sleep occurs cyclically throughout the night at intervals of approximately 90 minutes in all age groups (Figure 2). ${ }^{20,21}$

Related to the sleep-wake cycle, cortisol and IL-6 level fluctuates in a constant pattern which termed the circadian rhythm. It reaches the peak level at about $08.00-09.00 \mathrm{AM}$ and gradually decreases, the lowest level is around midnight; low cortisol during nighttime sleep is advantageous; the dysregulation of this circadian rhythm is able to disturb the sleep-wake cycle (Figure 3). ${ }^{22,23}$

\section{Cortisol values over a 24-hour period.}

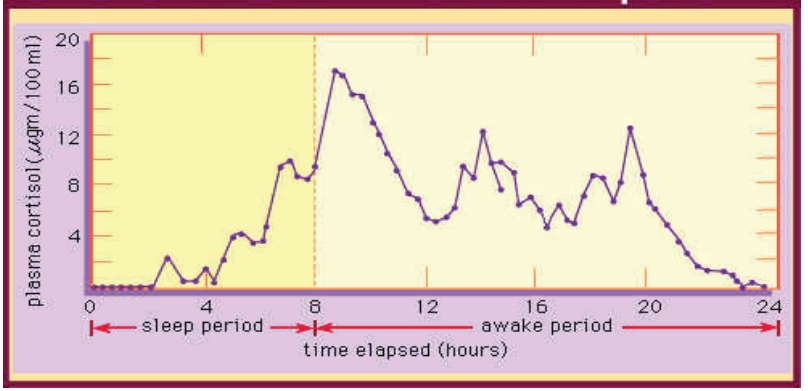

Figure 3. Cortisol level and the sleep - wake cycle over a 24-hour period. ${ }^{23}$

Sleep disturbances, in general are the manifestation of disrupted sleep-wake cycle; as a clinical syndrome is defined as difficulty in falling or staying asleep, or non refreshing sleep. ${ }^{14}$ Other criteria for sleep disturbances are: 1) insomnia: difficulty with falling asleep or staying asleep, 2) Sleep-onset insomnia: difficulty with falling asleep, 3) Sleep-maintenance insomnia: fragmented sleep, difficulty with maintaining sleep, 4) Sleep-disordered breathing (SDB): some degree of sleep-related upper airway obstruction, ranging in severity from upper airway resistance syndrome (UARS) to obstructive sleep apnea (OSA). ${ }^{3}$ Sleep disturbances may arise from several causes such as fear condition, aging, or disease, chronic fatigue 
syndrome; elevation of cortisol and interleukin-6 (IL-6) during night time. ${ }^{7,21,24,25}$

\section{Periodontal disease and sleep disturbances}

According a case report, sleep disturbances may have a correlation with periodontal disease. A female patient, aged 37, had been suffered from sleep disturbances, headache, musculoskeletal pains, fatigue and symptoms mimicking PMS and dysmenorrhoe for approximately one year. This patient also suffered from chronic periodontitis. After deep scaling and curettage had been done, the painful symptoms such as headache disappeared instantly; at the same night the patients also had an undisturbed sleep. Before and during her next menstrual period, the female patient did not suffered from painful symptoms and symptoms mimicking PMS. The patient was evaluated six month later and all symptoms did not reappear. ${ }^{10}$

\section{DISCUSSION}

According to literatures, the interrelationship of migraine headache, stress, sleep disturbances, female sexual hormones, PMS and dysmenorrhoe is exist. Nevertheless, which symptom initiates the chain reaction is still in controversy. According to Bolay et al. ${ }^{26}$ migraine headache is triggered by stress and sleep disturbance. Conversely, according to Baker et al. ${ }^{14}$ pains in dysmenorrhoe, PMS and headache lead to sleep disturbances. In addition, sleep disturbances may be triggered by elevated body temperature in the luteal phase that is mediated by progesterone.

In general, female sexual hormones play a role in the etiology of sleep disorders in women, either by having a direct effect on sleep processes or through their effect on mood and emotional state. Sexual hormones influence EEG sleep during the luteal phase by increasing the EEG frequency and core body temperature. It is also suggested that lack of estrogen, later in life, contributes to vasomotor symptoms, including hot flashes that cause sleep disturbances. Dysmenorrhoe is associated with significantly disturbed sleep quality prior to menses. When compared to control women, women with dysmenorrhoe had altered sex hormones, body temperature, and sleep throughout the menstrual cycle., , $^{2}$

Despite the controversial theories about the relationship between sleep disturbances and PMS; their treatment protocols are mostly indicated for anxiety, depression or hormonal regulation. As this condition could be worsen and lasting for months or years, another solution should be investigated to avoid unwanted effects of the medications. Fortunately, some case reports revealed that periodontal treatments that were scaling and curettage, which considered to be noninvasive, were able to relief pain, such as migraine, musculoskeletal pains and dysmenorrhoe; fatigue, sleep disturbances and other symptoms mimicking PMS. ${ }^{10,27}$

Periodontal disease is a potential source of bacterial endotoxins (lipopolysaccharides, LPS) from Gram-negative bacteria and pro-inflammatory cytokines interleukin-1
(IL-1), tumor necrosis factor- $\alpha$ (TNF- $\alpha$ ) and IL-6. The increase level of these pro-inflammatory cytokines could also be detected in blood serum. ${ }^{9}$ Current proposed mechanism that could elucidate how periodontal disease is able to initiate inflammation in distant site is the neurogenic switching mechanism. It is the interplay between immunogenic and neurogenic inflammation; through this mechanism, the maxillary nerve (CN V2) is able to elicit sinusitis and migraine symptoms via the sphenopalatine ganglion (SPG). ${ }^{28,29}$

In the reviewed case report, the first symptom that instantly disappeared after scaling procedure was headache, followed by sleep disturbance at the same night. It was relevant to articles by Boyd ${ }^{28}$ and Bolay et al. ${ }^{26}$ about the involvement of the trigeminal nerve in headache stimulation. Concurrently, it is also important that according to Bolay et al. ${ }^{26}$ migraine headache acts as an etiology of sleep disturbances. The possible explanation of the stimulation and the resolution of migraine headache which initiated by periodontal disease is as follows. First, the sensitization mechanism, headache in this case was primarily stimulated by pro-inflammatory mediators involved in chronic periodontal disease (i.e. IL-1, TNF- $\alpha$, IL-6 and PGE2), bradykinin and NO via the neurogenic switching mechanism. ${ }^{28-30}$

Neuropeptides which released by maxillary periodontal primary afferent sensory nerve fibers are able to sensitize the SPG which subsequently stimulate the vasodilatation of arteries in the meninges and elicits pain in the surrounding primary afferent sensory fibers. ${ }^{28}$ Secondly, the resolution or the cut off mechanism; as periodontal disease in this case report was considered as chronic periodontitis, deep scaling mostly followed by bleeding; this bleeding was considered advantageous because it facilitates drainage of pro-inflammatory mediators from the inflamed tissue. As the result, it reduced or cut off the neurogenic switching mechanism. ${ }^{30}$

Concerning about the susceptibility of women in inflammatory diseases, according to Nalbandian, low estrogen and physiological estrogen (17- $\alpha$ estradiol, E2) levels are stimulators of Th1 immune response that activate macrophages or monocytes. ${ }^{11}$ The activated macrophages by $\mathrm{E} 2$ are able to release additional pro-inflammatory cytokines i.e. IL-1, TNF- $\alpha$ and IL- 6 upon LPS challenge which stimulate the HPA-axis that leads to increase cortisol synthesis. Interleukin-6 is also renowned as a potential stimulator of HPA-axis an has often been called as stressinducible cytokine. ${ }^{31,32}$

The proposed mechanism of the relationship between chronic periodontitis and sleep disturbance, especially cortisol and IL-6 dependent sleep, could be explained as follows: chronic periodontitis leads to increase serum IL-6 level, therefore stimulates cortisol synthesis which in turn inhibits IL-6 level. If stress also involved, there will be additional increase of IL-6, which also followed by cortisol. Stages 3 and 4 that are deep sleep stages of the SWS during normal sleep-wake cycle in nighttime are associated with low IL-6 level and declining cortisol level. ${ }^{21}$ Consequently, 
high level of IL-6 and cortisol have adverse effects in the sleep-wake cycle because they lead to wakefulness and sleep disturbance. The repetition of IL-6 and cortisol stimulation-inhibition mechanism causes disturbance of sleep-wake cycle, as the result, the patient could not reach the deep sleep stage., $9,21,32$

Sleep disturbances also related to estrogen dominance which has nearly the same characteristics with PMS. ${ }^{3,19}$ While stimulated HPA-axis increase of cortisol synthesis, it may also decrease progesterone level in several ways: 1) as cortisol itself is a derivate of progesterone, increase of cortisol synthesis may lead to decrease of progesterone level, 2) by competitive binding; as cortisol and progesterone has the same affinity both to corticoid and progesterone receptors, cortisol may binds to progesterone receptors. These mechanisms may reduce the circulating progesterone level, thus increasing the estrogen level and cause estrogen dominance. ${ }^{16,18,19}$

In contrast to Baker et al. findings about the connection of the luteal phase, or the high progesterone phase, with sleep disturbances; the female patient in the reviewed case report suffered from headache and sleep disturbances everyday, albeit she was not having her premenstrual or menstrual period. So far, according to literatures in this review, women are more susceptible to stress, sexual hormonal imbalance, and periodontal diseases. Consequently, since periodontal disease is a potent source of LPS; stress and low and physiologic E2 which exposed to LPS challenged macrophages are able to produce additional pro-inflammatory cytokines, especially IL-6 that stimulate the HPA-axis. ${ }^{5,10,11,14,31}$ These mechanism is proposed to answer the question, why women also more vulnerable to sleep disturbances, especially which are cortisol and IL-6 dependent.

Since periodontal treatment was able to relief headache instantly and sleep disturbance at the same night, therefore it was strongly suggested that periodontal treatment eliminated the symptoms. How periodontal treatment, in this case deep scaling procedure and curettage, resulting in the disappearance of sleep disturbance could be explained in two ways: 1) deep scaling and curettage reduces the source of infection by removing subgingival biofilms, a potential source of LPS; 2) they also stimulate bleeding of the inflamed periodontal tissue, thus resulting in drainage of the pro-inflammatory mediators and cut off the neurogenic switching mechanism.

Resolution of periodontal inflammation which is the source of pro-inflammatory cytokines that responsible in sleep disturbances, especially IL-6, subsequently reduces stimulation of the HPA-axis, hence also regulate the cortisol circadian rhythm. Concerning to the disappearance of sleep disturbances and symptoms mimicking PMS before the next menstrual period after periodontal treatment in the reviewed case report; the conclusion is that chronic periodontitis may act as an etiology of sleep disturbances associated with PMS. Nevertheless, further researches are needed to include periodontal treatments in the treatment protocols of sleep disturbance and PMS.

\section{REFERENCES}

1. Kemp G, Segal R. Tips for a good night's sleep. Available online at URL http://www.helpguide.org/life/sleep tips.htm. Accessed December 3, 2005

2. National Center of Sleep Disorder Research. Sleep, sex differences, and women's health. Available online at URL http://www.nhlbi.nih. gov/ health/prof/sleep/res plan/section4/section4a.html. Accessed Nov 22, 2006

3. Hertz G, Cataletto ME. Sleep dysfunction in women. Available online at URL http://www.emedicine.com/neuro/topic656.htm Accessed November 2, 2006.

4. Moreno MA, Giesel A. Premenstrual syndrome (PMS). Updated May, 2006. Available online at URL http://www.emedicine.com/ ped/topic1890.htm. Accessed December 5, 2006.

5. Shansky RM, Bloom CG, Lerman D, McRae P, Benson C, Miller K, et al. Estrogen mediates sex differences in stress-induced prefrontal cortex dysfunction. Mol Psychiatry. 2004; 9(5):531-8.

6. Gremillion HA. Multidisciplinary diagnosis and management of orofacial pain. General Dentistry 2000; 2(1):178-84.

7. Afari N, Buchwald D. Chronic fatigue syndrome: a review. Am J Psychiatry 2003; 160(2):221-6

8. Güncü CN, Tözüm TF, Çağlayan F. Effects of endogenous sex hormones on the periodontium. Aus Dent Jour 2005; 50(3):138-45.

9. Lavigne SE. Your mouth-portal to your body. Probe 2004; 38(3): 114-34.

10. Utomo H, Prahasanti C. Periodontal disease as an etiology of orofacial and musculoskeletal pains in women. Indonesian Journal of Dentistry Special edition for KPPIKG UI XIV 2006; 13(2):202-5.

11. Nalbandian G, Kovats S. Estrogen, immunity and autoimmune disease. Curr Med Chem Immun Endocrinol Metab Agents. 2005; 5(1):85-91.

12. Dickerson LM, Mazyck PJ, Hunter MH. Premenstrual syndrome. Am Fam Phys 2003; 67(8):1743-52.

13. University of Maryland Medical Center. Premenstrual syndrome. Available online at URL http://www.umm.edu/women/pms.htm. Accessed December 5, 2006.

14. Baker CF, Driver HS, Paiker J, Rogers GG, Mitchell D. Acetaminophen does not affect 24-h body temperature of sleep in the luteal phase of the menstrual cycle. J Appl Physiol 2002; 92(4):1684-91.

15. Tostes RC, Nigro D, Fortes ZB, Carvalho MHC. Effects of estrogen on the vascular system. Braz J Med Biol Res 2003; 36(2):1143-58.

16. Van den Broek HH, Damoiseaux JG, De Baets MH, Hupperts RM. The influence of sex hormones on cytokines in multiple sclerosis and experimental autoimmune encephalomyelitis: a review. Multiple Sclerosis 2005; 11(3):349-59.

17. Goldman N, Glei DA, Seplaki C, Liu IW, Weinstein M. Perceived stress and physiological dysregulation in older adults. Stress 2005; 8(2):95-105

18. Quinckler M, Meyer B, Vogt CB, Grossmann C, Gruber U, et al. Agonistic and antagonistic properties of progesterone metabolites at the human mineralocorticoid receptor. Eur J Endocrinol 2002; 146:789-800.

19. Lam M. Estrogen Dominance: the silent epidemic Available online at URL. http://www.LamMD.com. Accessed November 12, 2005.

20. Sherwood L. Fundamentals of physiology : a human perspective. $3^{\text {rd }}$ ed. Belmont: Thomson Brooks/Cole; 2005. p. 133-35.

21. Vgontzas AN, Chrousos GP. Chapter 31. HPA axis and sleep. Endotext. 2005. Available at URL. http: // www.endotext.com. Accessed November 2, 2005.

22. Lemoyne college website. Stages of sleep and brain mechanisms. Available online at URL http://web.lemoyne.edu/ hevern/psy340/ graphics/sleep_cycle.jpg. Accessed.

23. Become healthy now. Cortisol circadian rhythm in the sleep and awake period. Available online at URL http://www.becomehealthynow. com/popups/circadian_rhythm.htm. Accessed November 20, 2006. 
24. Redwine L, Hauger RL, Gillin JC, Irwin M. Effects of sleep and sleep deprivation on interleukin-6, growth hormone, cortisol, and melatonin levels in humans. J Clin Endocrinol Metab 2000; 85(10):3597-603.

25. Alesci S, Martinez PE, Kelkar S, Ilias I, Ronsaville DS, Listwak SJ, et al. Major depression is associated with significant diurnal elevation in plasma interleukin- 6 levels, a shift of its circadian rhythm and loss of physiological in its secretion: clinical implications. J Clin Endocrinol Metabolism 2005; 90(5):2522-30

26. Bolay H, Reuter U, Dunn AK, Huang Z, Boas D. Intrinsic brain activity triggers trigeminal meningeal afferents in a migraine model. Nature Med 2002; 8(2):136-42.

27. Utomo H, Prahasanti C. Penyakit periodontal pada nyeri kepala dan nyeri haid. Edisi khusus Lustrum FKG Universitas Gadjah Mada, 2005; 14(4):101-6.

28. Boyd J. Pathophysiology of migraine and rationale for a targeted approach and prevention. Available online at URL http://www. migraineprevention.com/index/ html. Accessed February 15, 2006.
29. Lundy W, Linden R. Neuropeptides and neurogenic mechanism in oral and periodontal inflammation. Crit Rev Oral Biol 2004; 15(2):82-98.

30. Utomo $\mathrm{H}$. The sphenopalatine ganglion sensitization by periodontal inflammation: a possible etiology for headache and sinusitis in children. Majalah Kedokteran Gigi (Dental Journal) 2006; 39(2): 66-71.

31. Padgett DA, Glaser R. How stress influence the immune response. Trends in Immunol 2003 Aug; 24(8):4-8.

32. Kamma JJ, Giannopoulou C, Vasdekis VDS, Mombelli A. Cytokine profile in gingival crevicular fluid of aggressive periodontitis: influence of smoking and stress. J Clin Periodontol 2004 October; 31(10):894-902. 